\title{
Implicitization and Parametrization of Quadratic Surfaces with One Simple Base Point
}

\author{
Xuhui Wang Falai Chen Jiansong Deng \\ Department of Mathematics \\ University of Science and Technology of China \\ Hefei, Anhui, 230026, P.R. China \\ \{chenfl,dengjs\}@ustc.edu.cn
}

\begin{abstract}
This paper discusses implicitization and parametrization of quadratic surfaces with one simple base point. The key point to fulfill the conversion between the implicit and the parametric form is to compute three linearly independent moving planes which we call the weak $\mu$-basis of the quadratic surface. Beginning with the parametric form, it is easy to compute the weak $\mu$-basis, and then to find its implicit equation. Inversion formulas can also be obtained easily from the weak $\mu$-basis. For conversion from the implicit into the parametric form, we present a method based on the observation that there exists one self-intersection line on a quadratic surface with one base point. After computing the self-intersection line, we are able to derive the weak $\mu$-basis, from which the parametric equation can be easily obtained. A method is also presented to compute the self-intersection line of a quadratic surface with one base point.
\end{abstract}

\section{Categories and Subject Descriptors}

I.1.2 [Symbolic and Algebraic Manipulation]: Algorithms-Algebraic algorithms; I.3.5 [Computer Graphics]: Computational Geometry and Object Modeling - Curve, surface, solid, and object representations

\section{General Terms}

Algorithms

\section{Keywords}

Implicitization, moving plane, parametrization, weak $\mu$-basis

\section{INTRODUCTION}

In Computer Aided Geometric Design and Geometric Modelling, there are two complimentary ways of representing geometric objects: implicitly and parametrically, each of which has different advantages and disadvantages. It is valuable to have both representations at the same time. Thus conversion

Permission to make digital or hard copies of all or part of this work for personal or classroom use is granted without fee provided that copies are not made or distributed for profit or commercial advantage and that copies bear this notice and the full citation on the first page. To copy otherwise, to republish, to post on servers or to redistribute to lists, requires prior specific permission and/or a fee.

ISSAC'08, July 20-23, 2008, Hagenberg, Austria.

Copyright 2008 ACM 978-1-59593-904-3/08/07 ...\$5.00. between parametric and implicit form has been an interesting research topic for the past twenty years. The process of converting a parametric form into an implicit form is called implicitization, whereas the reverse process in called parameterization. In this paper, we are dedicated to investigating the implicitization and parametrization of a quadratic surface with one simple base point, which will be referred to as QSOB in the rest of the paper for brevity.

There is a wealth literature focusing on implicitization of parametric surfaces in the past two decades, and various methods have been proposed to solve the implicitization problem. These methods can be mainly classified into three categories: the resultant based method, the Groebner bases method and the method of moving surfaces (see [3, $9,16,17]$ and the references therein). The resultant based method is generally efficient. However, it fails in many situations, for example, in the presence of base points. On the other hand, the method based on Groebner bases is computational expensive. The method of moving surfaces proposed by Sederberg and the second author (of this paper) is generally much more efficient than the methods based on Groebner bases and resultants, and it even simplifies computation in the presence of base points. However, the method of moving surfaces is largely at a experimental stage, and there is no proof to show that the method of moving surfaces always works. A rigorous approach along this direction is a technique based on syzygies (or $\mu$-basis). The concept of $\mu$-basis was firstly introduced in [12] to provide a compact representation for the implicit equation of a rational curve. It was then generalized to rational ruled surfaces and general rational parametric surfaces $[5,6,8]$. Unfortunately, the $\mu$-basis doesn't directly provide an implicit equation for a general rational surface. In this paper, we will show that the method of moving surfaces works well for quadratic surfaces with one simple base point. Specifically, we will show that a QSOB has exactly three linearly independent moving planes which we call the weak $\mu$-basis of the QSOB. From the weak $\mu$-basis, the implicit equation of the QSOB can be easily derived.

Since the implicit degree of a QSOB is $2^{2}-1=3$, the implicit representation of a QSOB is a cubic surface. There are many works discussing the parametrization of nonsingular cubic surfaces $[1,2,18,20,21]$, and most of these approaches are based on the properties that a nonsingular cubic surface contains exactly 27 straight lines and 45 tritangent planes [14]. In [2], Berry and Patterson unified the parametrization and the implicitization of nonsingular cubic surfaces by using the Hilbert-Burch matrices, which is 
essentially another representation of three moving planes. However, the cubic surface derived from a QSOB is a singular surface, which doesn't have the above properties. In section 4, we will show that the cubic surface derived from a QSOB has a self-intersection line. Based on this observation, an algorithm is presented to derive a weak $\mu$-basis from the implicit equation of a QSOB, and thus a parametric equation is obtained.

The remainder of the paper is organized as follows. In Section 2, we recall some preliminary results about moving planes and cubic surfaces. In Section 3 and Section 4, we present techniques for the implicitization and the parametrization of a QSOB. Singularities of a QSOB are also analyzed in detail. We conclude the paper in Section 5.

\section{PRELIMINARIES}

Let $R[s, t]$ be the ring of bivariate polynomials in $s, t$ over some infinite field $F$. A rational parametric surface in homogeneous form is defined as follows:

$$
\mathbf{P}(s, t)=(a(s, t), b(s, t), c(s, t), d(s, t)),
$$

where $a, b, c, d \in R[s, t]$ are polynomials with $\operatorname{gcd}(a, b, c, d)=$ 1.

A base point of a rational surface $\mathbf{P}(s, t)$ is a parameter pair $\left(s_{0}, t_{0}\right)$ such that $\mathbf{P}\left(s_{0}, t_{0}\right)=(0,0,0,0)$. Here $s_{0}$ and $t_{0}$ lie in the algebraically closed extension filed of $F$. A QSOB is a rational quadratic parametric surface with one simple base point. The total degree of a QSOB in $s, t$ is 2 and its implicit degree is $2^{2}-1=3$. In this paper, we will provide an efficient and rigorous method for implicitization and parametrization of a QSOB using the method of moving planes.

A moving plane is a family of planes with parameter pair $(s, t)$ :

$$
L(\mathbf{X} ; s, t):=A(s, t) x+B(s, t) y+C(s, t) z+D(s, t) w=0,
$$

where $\mathbf{X}=(x, y, z, w)$ and $A(s, t), B(s, t), C(s, t), D(s, t) \in$ $R[s, t]$. Sometimes, we write it in a vector form: $\mathbf{L}(s, t):=$ $(A(s, t), B(s, t), C(s, t), D(s, t))$. A moving plane $\mathbf{L}(s, t)$ is said to follow the rational surface $\mathbf{P}(s, t)$ if

$$
\begin{aligned}
\mathbf{L}(s, t) \cdot \mathbf{P}(s, t)= & a(s, t) A(s, t)+b(s, t) B(s, t)+ \\
& c(s, t) C(s, t)+d(s, t) D(s, t) \equiv 0 .
\end{aligned}
$$

For a QSOB, there are exactly three independent moving planes of degree 1 in $s, t$, and these three moving planes serve as the connection between the parametric form and the implicit form of the QSOB.

Since the implicit degree of a QSOB is three, the following result is useful for analyzing the singularities of QSOBs.

Proposition 2.1. [15] If a cubic surface has finitely many singular points, then the number of singular points is less than or equal to four. Moreover, the cubic surface containing precisely 4 singular points is unique up to linear isomorphism.

\section{IMPLICITIZATION}

In this section, we temporarily switch the parameters $s, t$ to homogeneous parameters $s, t, u$. Then the parametric surface $\mathbf{P}(s, t)$ becomes

$$
\mathbf{P}(s, t, u)=(a(s, t, u), b(s, t, u), c(s, t, u), d(s, t, u)) .
$$

For a quadratic surface $\mathbf{P}(s, t, u)$ with one base point $\left(s_{0}\right.$, $\left.t_{0}, u_{0}\right) \neq(0,0,0)$, there exist a nonsingular matrix $B \in$ $F^{3 \times 3}$ such that $\left(s_{0}, t_{0}, u_{0}\right)=(0,1,0) \cdot B$. Let $(s, t, u)=$ $(\tilde{s}, \tilde{t}, \tilde{u}) \cdot B$, then we get a new parametric representation for the quadratic surface:

$$
\tilde{\mathbf{P}}(\tilde{s}, \tilde{t}, \tilde{u})=(\tilde{a}(\tilde{s}, \tilde{t}, \tilde{u}), \tilde{b}(\tilde{s}, \tilde{t}, \tilde{u}), \tilde{c}(\tilde{s}, \tilde{t}, \tilde{u}), \tilde{d}(\tilde{s}, \tilde{t}, \tilde{u}))
$$

which has the same implicit representation as $\mathbf{P}(s, t, u)$.

Since $\tilde{\mathbf{P}}(\tilde{s}, \tilde{t}, \tilde{u})$ contains the base point $(0,1,0)$, it doesn't contain the term $t^{2}$. Therefore, without loss of generality, we may assume that, in non-homogeneous variables, the quadratic surface $\mathbf{P}(s, t)$ has the form

$$
\mathbf{P}(s, t)=\mathbf{P}_{1}^{T}+\mathbf{P}_{s}^{T} s+\mathbf{P}_{t}^{T} t+\mathbf{P}_{s^{2}}^{T} s^{2}+\mathbf{P}_{s t}^{T} s t,
$$

where $\mathbf{P}_{1}, \mathbf{P}_{s}, \mathbf{P}_{t}, \mathbf{P}_{s t}$ and $\mathbf{P}_{s^{2}}$ are all column vectors in $F^{4}$.

Before going on, we give a simple method to detect if a given quadratic parametrization is a QSOB or not. Let

$$
\left\{\begin{array}{l}
A_{1} x+B_{1} y+C_{1} z+D_{1}=0 \\
A_{2} x+B_{2} y+C_{2} z+D_{2}=0
\end{array}\right.
$$

be an arbitrary line. The intersection of this line with the parametric equation (1) of a QSOB is determined by

$$
\left\{\begin{array}{l}
f(s, t):=A_{1} a(s, t)+B_{1} b(s, t)+C_{1} c(s, t)+D_{1} d(s, t)=0 \\
g(s, t):=A_{2} a(s, t)+B_{2} b(s, t)+C_{2} c(s, t)+D_{2} d(s, t)=0
\end{array}\right.
$$

Let $h$ be the resultant of $f$ and $g$ with respect to $s$ (or $t$ ). If $\operatorname{deg}(h)=3$, then the quadratic parametrization is a QSOB.

A major observation about QSOBs is the following theorem.

THEOREM 3.1. For a QSOB, there are exactly three linearly independent moving planes with degree one in $s, t$ that follow it.

ProOF. Let

$$
\mathbf{L}(s, t)=\mathbf{L}_{s} s+\mathbf{L}_{t} t+\mathbf{L}_{1}
$$

be a moving plane with total degree 1 in $s, t$ that follows $\mathbf{P}(s, t)$. Here $\mathbf{L}_{1}, \mathbf{L}_{s}$ and $\mathbf{L}_{t}$ are row vectors in $F^{4}$.

From

$$
\mathbf{L}(s, t) \cdot \mathbf{P}(s, t) \equiv 0,
$$

one obtains

$$
\left(\mathbf{L}_{s}, \mathbf{L}_{t}, \mathbf{L}_{1}\right) \cdot Q=\mathbf{0},
$$

where $Q$ is the $12 \times 9$ matrix

$$
Q=\left(\begin{array}{ccccccccc}
\mathbf{0} & \mathbf{P}_{1} & \mathbf{0} & \mathbf{P}_{s} & \mathbf{P}_{t} & \mathbf{0} & \mathbf{P}_{s^{2}} & \mathbf{P}_{s t} & \mathbf{0} \\
\mathbf{0} & \mathbf{0} & \mathbf{P}_{1} & \mathbf{0} & \mathbf{P}_{s} & \mathbf{P}_{t} & \mathbf{0} & \mathbf{P}_{s^{2}} & \mathbf{P}_{s t} \\
\mathbf{P}_{1} & \mathbf{P}_{s} & \mathbf{P}_{t} & \mathbf{P}_{s^{2}} & \mathbf{P}_{s t} & \mathbf{0} & \mathbf{0} & \mathbf{0} & \mathbf{0}
\end{array}\right)
$$

We first note that the rank of the set of vectors $\left\{\mathbf{P}_{1}, \mathbf{P}_{s}\right.$, $\left.\mathbf{P}_{t}, \mathbf{P}_{s^{2}}, \mathbf{P}_{s t}\right\}$ must be four, otherwise the implicit representation of $\mathbf{P}(s, t)$ is a plane. Now we claim that $\operatorname{rank}(Q)=9$, which would imply that there are exactly three linearly independent moving planes of degree one in $s, t$ following $\mathbf{P}(s, t)$.

Suppose on the contrary that the columns $\mathbf{C}_{i}$ of $Q$ are linearly dependent. Then there exist constants $k_{1}, k_{2} \ldots, k_{9}$, 
at least one of them are nonzero, such that

$$
\sum_{i=1}^{9} k_{i} \mathbf{C}_{i}=\mathbf{0}
$$

The above equation is equivalent to

$$
\left(\mathbf{P}_{1}, \mathbf{P}_{s}, \mathbf{P}_{t}, \mathbf{P}_{s^{2}}, \mathbf{P}_{s t}\right) \cdot K=\mathbf{0}
$$

where

$$
K=\left(\begin{array}{lll}
k_{1} & k_{2} & k_{3} \\
k_{2} & k_{4} & k_{5} \\
k_{3} & k_{5} & k_{6} \\
k_{4} & k_{7} & k_{8} \\
k_{5} & k_{8} & k_{9}
\end{array}\right)
$$

Since $\operatorname{rank}\left(\mathbf{P}_{1}, \mathbf{P}_{s}, \mathbf{P}_{t}, \mathbf{P}_{s^{2}}, \mathbf{P}_{s t}\right)=4$, we conclude that $\operatorname{rank}(K)=1$. We assume that at least one of $k_{1}, k_{2}, k_{3}, k_{4}, k_{5}$ is nonzero (the other cases can be treated similarly and hence are omitted), then there exist constants $\lambda$ and $\mu$ such that

$$
\begin{aligned}
& k_{2}=\lambda k_{1}, \quad k_{4}=\lambda k_{2}, \quad k_{5}=\lambda k_{3}, \\
& k_{7}=\lambda k_{4}, \quad k_{8}=\lambda k_{5}, \\
& k_{3}=\mu k_{1}, \quad k_{5}=\mu k_{2}, \quad k_{6}=\mu k_{3}, \\
& k_{8}=\mu k_{4}, \quad k_{9}=\mu k_{5} .
\end{aligned}
$$

Hence

$$
k_{1} \neq 0, \quad k_{2}=\lambda k_{1}, \quad k_{3}=\mu k_{1}, \quad k_{4}=\lambda^{2} k_{1}, \quad k_{5}=\lambda \mu k_{1} .
$$

Therefore

$$
\mathbf{P}_{1}+\lambda \mathbf{P}_{s}+\mu \mathbf{P}_{t}+\lambda^{2} \mathbf{P}_{s^{2}}+\lambda \mu \mathbf{P}_{s t}=\mathbf{0},
$$

which means that $\mathbf{P}(s, t)$ has another base point $(\lambda, \mu, 1)$ apart from $(0,1,0)$, a contradiction. Hence, $\operatorname{rank}(Q)=9$ and there exist three linearly independent moving planes following $\mathbf{P}(s, t)$.

REMARK 3.1. The three moving planes in Theorem 3.1 are called a weak $\mu$-basis of the QSOB. Since a weak $\mu$-basis corresponds to a basis of the solution space of the linear system of equations (5), two different weak $\mu$-bases differ by the multiplication of a $3 \times 3$ invertible matrix. Therefore, the weak $\mu$-basis of a QSOB is unique in the sense that it is the same under an invertible linear transformation.

The weak $\mu$-basis provides a direct method to implicitize a QSOB.

THEOREM 3.2. Let

$$
L_{i}(\mathbf{X} ; s, t)=L_{i 1}(\mathbf{X}) s+L_{i 2}(\mathbf{X}) t+L_{i 3}(\mathbf{X}),
$$

$i=1,2,3$ be a weak $\mu$-basis of $\mathbf{P}(s, t)$. Here $L_{i j}(\mathbf{X})$ is a linear function in $x, y, z, w, i, j=1,2,3$. Then the implicit equation of $\mathbf{P}(s, t)$ is given by $f(\mathbf{X}):=\operatorname{det}(M)=0$. Here

$$
M=\left(\begin{array}{lll}
L_{11}(\mathbf{X}) & L_{12}(\mathbf{X}) & L_{13}(\mathbf{X}) \\
L_{21}(\mathbf{X}) & L_{22}(\mathbf{X}) & L_{23}(\mathbf{X}) \\
L_{31}(\mathbf{X}) & L_{32}(\mathbf{X}) & L_{33}(\mathbf{X})
\end{array}\right)
$$

Proof. Firstly, we will prove that the three moving planes $L_{1}, L_{2}$ and $L_{3}$ are $R[x, y, z, w]$-linearly independent, or equivalently, $f(\mathbf{X})$ is not identically zero.

We first point out several basic facts. (i) Let $Q=\left(q_{i j}\right)_{4 \times 4}$ be an invertible matrix, and let

$$
\begin{array}{r}
\tilde{\mathbf{P}}(s, t):=(\tilde{a}(s, t), \tilde{b}(s, t), \tilde{c}(s, t), \tilde{d}(s, t))^{T} \\
=Q(a(s, t), b(s, t), c(s, t), d(s, t))^{T} .
\end{array}
$$

Suppose that the implicit equation of $\tilde{\mathbf{P}}(s, t)$ is $\tilde{f}(\mathbf{X})=$ 0 . Then the implicit equation of $\mathbf{P}(s, t)$ is $\tilde{f}\left(\mathbf{X} \cdot Q^{T}\right)=$ 0 .

(ii) $\mathbf{P}_{1}, \mathbf{P}_{t}, \mathbf{P}_{s t}$ and $\mathbf{P}_{s^{2}}$ are all non-zero vectors. In fact, $\mathbf{P}_{t}=\mathbf{0}$ or $\mathbf{P}_{s t}=\mathbf{0}$ indicates that $(0,1,0)$ is a double base point of $\mathbf{P}(s, t) . \mathbf{P}_{1}=\mathbf{0}$ implies $(0,0,1)$ is a base point of $\mathbf{P}(s, t)$. Similarly, $\mathbf{P}_{s^{2}}=\mathbf{0}$ means $(1,0,0)$ is also a base point of $\mathbf{P}(s, t)$. But $\mathbf{P}(s, t)$ has only one base point $(0,1,0)$.

(iii) By elementary row operations, the $4 \times 5$ matrix $\left(\mathbf{P}_{1}, \mathbf{P}_{s}\right.$, $\left.\mathbf{P}_{t}, \mathbf{P}_{s^{2}}, \mathbf{P}_{s t}\right)$ can be reduced into

$$
\left(\tilde{\mathbf{P}}_{1}, \tilde{\mathbf{P}}_{s}, \tilde{\mathbf{P}}_{t}, \tilde{\mathbf{P}}_{s^{2}}, \tilde{\mathbf{P}}_{s t}\right)=\left(\begin{array}{ccccc}
1 & e_{1} & 0 & 0 & 0 \\
0 & e_{2} & 1 & 0 & 0 \\
0 & e_{3} & 0 & 1 & 0 \\
0 & e_{4} & 0 & 0 & 1
\end{array}\right)
$$

(iv) $e_{1} e_{3}-1=0$ and $e_{1} e_{4}+e_{2}=0$ can't hold simultaneously. Here $e_{i}, i=1,2,3,4$ are elements in the matrix (8). Indeed, suppose towards contradiction that both equalities hold. Then the parametric surface

$$
\tilde{\mathbf{P}}(s, t)=\tilde{\mathbf{P}}_{1}+\tilde{\mathbf{P}}_{s} s+\tilde{\mathbf{P}}_{t} t+\tilde{\mathbf{P}}_{s^{2}} s^{2}+\tilde{\mathbf{P}}_{s t} s t
$$

has a base point $\left(-1, e_{2}, e_{1}\right)$, which contradicts the fact that $\tilde{\mathbf{P}}(s, t)$ has just one base point.

Now we may assume that $e_{1} e_{3}-1 \neq 0$ (the case when $e_{1} e_{4}+e_{2} \neq 0$ is similar and hence is omitted). One can easily compute that a weak $\mu$-basis of $\tilde{\mathbf{P}}(s, t)$ is

$$
\begin{aligned}
& L_{1}(\mathbf{X}):=\beta z-\alpha w+(-\gamma x+\alpha y) s, \\
& L_{2}(\mathbf{X}):=-\alpha y+\beta e_{1} z-\alpha e_{1} w-\beta x s+\alpha x t, \\
& L_{3}(\mathbf{X}):=e_{4} z-e_{3} w-w s+z t,
\end{aligned}
$$

where $\alpha=e_{1} e_{3}-1, \beta=e_{1} e_{4}+e_{2}, \gamma=e_{2} e_{3}+e_{4}$. It can be easily seen that

$$
f(\mathbf{X}):=\left|\begin{array}{ccc}
-\gamma x+\alpha y & 0 & \beta z-\alpha w \\
-\beta x & \alpha x & -\alpha y+\beta e_{1} z-\alpha e_{1} w \\
-w & z & e_{4} z-e_{3} w
\end{array}\right|
$$

contains the term $\alpha^{2} y^{2} z$. Thus $f(\mathbf{X}) \not \equiv 0$.

Secondly, for any parameter values $\left(s_{0}, t_{0}\right)$, let $\mathbf{X}_{0}=$ $\left.\left(a\left(s_{0}, t_{0}\right), b\left(s_{0}, t_{0}\right)\right), c\left(s_{0}, t_{0}\right), d\left(s_{0}, t_{0}\right)\right)$. We want to show that $f\left(\mathbf{X}_{0}\right)=0$. From $L_{i}\left(\mathbf{X}_{0}, s_{0}, t_{0}\right)=0, i=1,2,3$, we have

$$
\left(\begin{array}{ccc}
L_{11}\left(\mathbf{X}_{0}\right) & L_{12}\left(\mathbf{X}_{0}\right) & L_{13}\left(\mathbf{X}_{0}\right) \\
L_{21}\left(\mathbf{X}_{0}\right) & L_{22}\left(\mathbf{X}_{0}\right) & L_{23}\left(\mathbf{X}_{0}\right) \\
L_{31}\left(\mathbf{X}_{0}\right) & L_{32}\left(\mathbf{X}_{0}\right) & L_{33}\left(\mathbf{X}_{0}\right)
\end{array}\right)\left(\begin{array}{c}
s_{0} \\
t_{0} \\
1
\end{array}\right)=\mathbf{0} .
$$

Therefore,

$$
f\left(\mathbf{X}_{0}\right)=0 .
$$

Since the degree of $f(\mathbf{X})$ is three, we can conclude that $f(\mathbf{X})=0$ is the implicit form of $\mathbf{P}(s, t)$.

REMARK 3.2. In [4], the authors proved that the moving planes and moving quadrics method works for general rational surfaces in the presence of base points if the rational surfaces satisfy certain conditions (BP1-BP5). It can be shown 
that a QSOB satisfies the BP1-PB5 conditions. Thus Theorem 3.2 can be looked as a special case of Theorem 3.6 in the paper [4].

Based on the results of Theorem 3.1 and 3.2, a simple algorithm can be devised to implicitize a QSOB.

\section{Algorithm IMPLICITIZATION-QSOB \\ Input: The parametric equation of a QSOB. \\ Output: The implicit eqaution of the QSOB. \\ Procedure:}

1. Solve the linear system of equations (5) to obtain the weak $\mu$-basis of the QSOB.

2. Compute the determinant of the matrix (7) formed by the weak $\mu$-basis. The determinant gives the implicit equation of the QSOB.

REMARK 3.3. To improve the efficiency, one may first perform row reductions on the matrix $\left(\mathbf{P}_{1}, \mathbf{P}_{s}, \mathbf{P}_{t}, \mathbf{P}_{s^{2}}, \mathbf{P}_{s t}\right)$ and transform it into the simplest form, i.e., four of the column vectors are transformed into $(1,0,0,0)^{T},(0,1,0,0)^{T}$, $(0,0,1,0)^{T}$ and $(0,0,0,1)^{T}$. Then we compute the weak $\mu$ basis and the implicit equation of the corresponding surface $\tilde{\mathbf{P}}(s, t)$ after the transformation. The implicit equation of the surface $\mathbf{P}(s, t)$ is obtained by the same transformation of the variables from the implicit equation of $\tilde{\mathbf{P}}(s, t)$.

We illustrate the algorithm with an example.

Example 1. Let the quadratic parametric surface be

$$
\mathbf{P}(s, t)=\left(s, t^{2}, s t-3, s-s t\right)
$$

with $(1,0,0)$ being a base point. A weak $\mu$-basis of $\mathbf{P}(s, t)$ is

$$
\left(L_{1}, L_{2}, L_{3}\right)=(s, t, 1)\left(\begin{array}{ccc}
0 & x-z-w & y \\
-x & -3 x & -x+w \\
x-w & -3 w & 0
\end{array}\right) .
$$

Then the implicit equation of $\mathbf{P}(s, t)$ is

$$
\begin{aligned}
& f(\mathbf{X}):=\left|\begin{array}{ccc}
0 & x-z-w & y \\
-x & -3 x & -x+w \\
x-w & -3 w & 0
\end{array}\right| \\
& =-x^{3}+3 x^{2} y+x^{2} z+3 x^{2} w-2 x z w-3 x w^{2} \\
& +z w^{2}+w^{3}=0 .
\end{aligned}
$$

The weak $\mu$-basis also provides a simple inversion formula for a QSOB. Suppose $\mathbf{X}_{0}$ is a point on a QSOB, the corresponding parameter values $\left(s_{0}, t_{0}\right)$ can be solved from $(10)$ :

$$
\left\{\begin{array}{l}
s_{0}=\frac{L_{12}\left(\mathbf{X}_{0}\right) L_{23}\left(\mathbf{X}_{0}\right)-L_{13}\left(\mathbf{X}_{0}\right) L_{22}\left(\mathbf{X}_{0}\right)}{L_{11}\left(\mathbf{X}_{0}\right) L_{22}\left(\mathbf{X}_{0}\right)-L_{12}\left(\mathbf{X}_{0}\right) L_{21}\left(\mathbf{X}_{0}\right)}, \\
t_{0}=\frac{L_{13}\left(\mathbf{X}_{0}\right) L_{21}\left(\mathbf{X}_{0}\right)-L_{11}\left(\mathbf{X}_{0}\right) L_{23}\left(\mathbf{X}_{0}\right)}{L_{11}\left(\mathbf{X}_{0}\right) L_{22}\left(\mathbf{X}_{0}\right)-L_{12}\left(\mathbf{X}_{0}\right) L_{21}\left(\mathbf{X}_{0}\right)} .
\end{array}\right.
$$

For the QSOB in Example 1, the inversion formula is

$$
s=\frac{3 x}{x-z-w}, \quad t=\frac{x-w}{x} .
$$

The weak $\mu$-basis has some nice algebraic properties. For example, one has
Theorem 3.3. Let $L_{i}(\mathbf{X}, s, t), i=1,2,3$ be a weak $\mu$ basis of a $Q S O B \mathbf{P}(s, t)$, and $f(\mathbf{X})=0$ be the implicit equation of $\mathbf{P}(s, t)$. Then

$$
\left\langle L_{1}, L_{2}, L_{3}\right\rangle \cap R[x, y, z, w]=\langle f\rangle .
$$

Here $R[x, y, z, w]$ is the ring of polynomials in variables $x, y$, $z, w$ over the field $F$.

Proof. Let $\left(h_{1}, h_{2}, h_{3}\right)=\left(L_{12}, L_{22}, L_{32}\right) \times\left(L_{13}, L_{23}, L_{33}\right)$. By Theorem 3.2, it is easy to show that $f=h_{1} L_{1}+h_{2} L_{2}+$ $h_{3} L_{3}$. Hence $f \in\left\langle L_{1}, L_{2}, L_{3}\right\rangle$.

On the other hand, for any $g(x, y, z, w) \in\left\langle L_{1}, L_{2}, L_{3}\right\rangle \cap$ $R[x, y, z, w]$, there exist polynomials $h_{i}, i=1,2,3$ such that

$$
g=h_{1} L_{1}+h_{2} L_{2}+h_{3} L_{3} .
$$

Since $L_{i}, i=1,2,3$ are moving planes following $\mathbf{P}(s, t)$, $g(a, b, c, d) \equiv 0$, i.e., $g$ vanishes on $\mathbf{P}(s, t)$. Since $f$ also vanishes on $\mathbf{P}(s, t)$ and it is irreducible, $f \mid g$. Hence $g \in\langle f\rangle$. This completes the proof.

\section{PARAMETRIZATION}

A QSOB represents a cubic surface in implicit form. Compared to non-singular cubic surfaces parameterized by cubic parametric surfaces with six generic base points, it has some special properties.

\subsection{Singular locus of a QSOB}

We first give two simple lemmas regarding the singular points of a cubic surface.

Lemma 4.1. Let $P_{1}$ and $P_{2}$ be two singular points of a cubic surface. Then the line joining the two singular points is contained in the surface.

Proof. Notice that any line intersects with a cubic surface at three points if the line is not contained in the cubic surface. Since the line joining $P_{1}$ and $P_{2}$ intersects with the cubic surface at four points (counting multiplicity), the line must be contained in the surface.

Lemma 4.2. An irreducible cubic surface either has a selfintersection line, or has at most four singular points.

Proof. If the cubic surface contains a non-planar space curve as its singular locus, then the line joining any two points on the space curve is contained in the surface by Lemma (4.1). Consequently some three-dimensional set is contained in the surface, an impossibility.

If the cubic surface contains a plane curve (not a line) as its locus, then the plane containing the plane curve is a part of the surface. This is impossible since the cubic surface is irreducible.

If the cubic surface contains a line $l$ and a point $P$ not on the line as its singular locus, then the plane determined by the point $P$ and the line $l$ is also contained in the surface, again a contradiction with the irreducibility of the cubic surface.

Finally, if the surface has only finite number of singular points, it has at most four singular points by Proposition 2.1 .

THEOREM 4.3. [10] The cubic surface parametrized by a QSOB is a singular surface. The singular locus is a selfintersection line. 
Proof. Coffman et al. ([10]) provide a proof based on matrix algebra. In this paper, we give a simple proof by using the weak $\mu$-basis of the QSOB.

Without of loss of generality, we assume that the quadratic parametric surface has the simplified form (9). Let $\tilde{M}$ be the matrix formed by the weak $\mu$-basis of $\tilde{\mathbf{P}}(s, t)$, i.e.,

$$
\tilde{M}:=\left(\begin{array}{ccc}
-\gamma x+\alpha y & 0 & \beta z-\alpha w \\
-\beta x & \alpha x & -\alpha y+\beta e_{1} z-\alpha e_{1} w \\
-w & z & e_{4} z-e_{3} w
\end{array}\right) .
$$

It is easy to show that $\operatorname{rank}(\tilde{M})=1$ if and only if $\beta z-$ $\alpha w=0$ and $-\gamma x+\alpha y=0$. That is, for any point $(x, y, z, w)$ satisfying the two equations, it corresponds to at least two parameters, and thus corresponds to a singular point. So the intersection line of two planes $\beta z-\alpha w=0$ and $-\gamma x+\alpha y=0$ is a double line of $\mathbf{P}(s, t)$. By Lemma 4.2, there are no other singular points on $\mathbf{P}(s, t)$.

Based on the proof of Theorem 4.3, a simple method is derived to compute the self-intersection line of a QSOB parameterized by $\mathbf{P}(s, t)$.

\section{Algorithm SELF-INTERSECTION-QSOB-I Input: The parametric equation $\mathbf{P}(s, t)$ of a QSOB. Output: The self-intersection line of the QSOB. Procedure:}

1. Compute the base point of $\mathbf{P}(s, t)$, and change the parametric equation into the form (4).

2. Compute a weak $\mu$-basis $L_{i}(\mathbf{X})=L_{i 1}(\mathbf{X}) s+L_{i 2}(\mathbf{X}) t+$ $L_{i 3}(\mathbf{X}), i=1,2,3$ of $\mathbf{P}(s, t)$.

3. Perform row operations to the matrix $M$ as defined in (7), such that one element of the second column is zero (say $L_{12}(\mathbf{X})=0$ ). This can happen because $L_{i 2}\left(\mathbf{P}_{t}\right)=0$ and $L_{i 2}\left(\mathbf{P}_{s t}\right)=0, i=1,2,3$. We still use the same notation for the matrix after row reduction.

4. The intersection of the two planes $L_{11}(\mathbf{X})=0$ and $L_{13}(\mathbf{X})=0$ is the self-intersection line of $\mathbf{P}(s, t)$.

For the quadratic parametric surface in Example 1, the self-intersection line is defined by $x-w=0$ and $x=0$, or equivalently $x=0$ and $w=0$.

For a QSOB defined by an implicit equation $f(x, y, z, w)$, how does one compute its self-intersection line? A standard way is to solve the system of equations:

$$
\begin{aligned}
& f_{x}(x, y, z, w)=0, \quad f_{y}(x, y, z, w)=0, \\
& f_{z}(x, y, z, w)=0, \quad f_{w}(x, y, z, w)=0 .
\end{aligned}
$$

Or equivalently, to compute the intersection of four quadric surfaces. Computing the intersection of two quadric surfaces is not an easy task (see [22] and the references therein). In the following, we provide a method to detect if an irreducible cubic surface has a self-intersection line, and if it has, how to compute the self-intersection line.

We consider the intersection of the cubic surface with an arbitrary plane. First, we have the following lemma.
Lemma 4.4. Let $P$ be a singular point of a cubic surface, and $\pi$ be a plane passing through $P$. Then $P$ is also a singular point of the curve which is the intersection of the cubic surface and the plane $\pi$.

ProOF. Without loss of generality, we may assume that the plane $\pi$ is defined by the equation $z=p_{1} x+p_{2} y+$ $p_{3} w$. Then the intersection curve of the plane $\pi$ and the cubic surface $f(x, y, z, w)=0$ is defined by $g(x, y, w):=$ $f\left(x, y, p_{1} x+p_{2} y+p_{3} w, w\right)=0$. If $P$ is a singular point of the cubic surface, then $f_{x}(P)=f_{y}(P)=f_{z}(P)=f_{w}(P)=$ 0 . So $g_{x}(P)=f_{x}(P)+f_{z}(P) p_{1}=0$. Similarly, $g_{y}(P)=$ $g_{w}(P)=0$. Thus $P$ is also a singular point of $g(x, y, w)=$ 0 .

Note that the converse of the above lemma is not true.

Now based on previous analysis, we can devise an algorithm to detect and compute the self-intersection line of a cubic surface.

\section{Algorithm SELF-INTERSECTION-QSOB-II}

Input: Implicit equation of an irreducible cubic surface.

Output: Detect if the cubic surface has a self-intersection line, and if yes, compute it.

Procedure:

1. Choose five random planes, and compute the intersection curves of the cubic surface with the five planes.

2. Compute the singular point (if any) of each intersection curve by the method in [7].

3. Verify if the singular points of the intersection curves are singular points of the cubic surface;

4. If we obtain 5 or more singular points of the cubic surface, then these singular points are all on a single line, and the line is the self-intersection line of the cubic surface. Otherwise, the cubic surface doesn't have a self-intersection line.

REMARK 4.1. To detect if a cubic surface is a QSOB or not, one can simply find a random plane to intersect the surface, and check if the intersect curve contains a singular point or not.

\subsection{Parametrization method}

Suppose we are given the implicit equation $f(x, y, z, w)=$ 0 of a cubic surface parameterized by a QSOB. After we have computed the self-intersection line, a direct method to parameterize the surface is as follows. We find an arbitrary plane passing through the self-intersection line, and the plane intersects the cubic surface at a line $l^{\prime}$ besides the self-intersection line. The line $l^{\prime}$ gives a parametrization of the cubic surface. Unfortunately, such a parametrization is in general cubic instead of quadratic. To get a quadratic parametrization, we would like to seek the aid of a weak $\mu$-basis.

To begin with, we choose two distinct planes $p_{1}=0, p_{2}=$ 0 passing through the self-intersection line $l$ of the cubic surface. The plane $p_{i}=0$ intersects with the cubic surface at $l$ (a double line) and another line $l_{i}, i=1,2$. Thus, if we solve for one variable (say $z$ ) from $p_{1}=0$ to get $z=$ 
$z_{1}(x, y, w)$, and substitute it into $f(x, y, z, w)$, then one gets $f\left(x, y, z_{1}(x, y, w), w\right)=m^{2} n_{1}$. Here $m, n_{1}$ are linear functions in $x, y, w$. Similarly, $f\left(x, y, z_{2}(x, y, w), w\right)=m^{2} n_{2}$. Here $z_{2}(x, y, w)$ is solved from $p_{2}=0$ and $n_{2}$ is a linear function in $x, y, w$.

TheOREM 4.5. Let the notation be as above. Then the implicit equation of the cubic surface parameterized by a $Q S O B$ can be written as $f(x, y, z, w)=\operatorname{det}(G)$, where

$$
G=\left(\begin{array}{ccc}
p_{1} & 0 & m \\
0 & p_{2} & m \\
-k_{1} n_{1} & -k_{2} n_{2} & h_{0}(x, y, z, w)
\end{array}\right)
$$

$k_{1}, k_{2}$ are undetermined coefficients, and $h_{0}(x, y, z, w)$ is a undetermined linear function.

Proof. The proof is similar to the proof of Theorem 5 in [2]. We sketch it as follows.

Since $f(x, y, z, w)$ vanishes on the self-intersection line $l$ and the other two lines $l_{1}, l_{2}, f(x, y, z, w)$ must be contained in the radical ideal

$$
J=\left\langle p_{1}, p_{2}\right\rangle \cap\left\langle p_{1}, n_{1}\right\rangle \cap\left\langle p_{2}, n_{2}\right\rangle .
$$

It is easy to show that

$$
J=\left\langle p_{1} p_{2}, p_{1} n_{2}, p_{2} n_{1}\right\rangle .
$$

So $f(x, y, z, w)$ can be expressed as

$$
h_{0} p_{1} p_{2}+h_{1} p_{1} n_{2}+h_{2} p_{2} n_{1},
$$

where $h_{0}, h_{1}, h_{2}$ are homogeneous polynomials in $R[x, y, z, w]$.

Next from $f(x, y, z, w) \in\left\langle p_{1}, m\right\rangle \cap\left\langle p_{1}, n_{1}\right\rangle$, one obtains that $m \mid h_{2}$. Similarly, we can get that $m \mid h_{1}$. Thus

$$
f(x, y, z, w)=h_{0} p_{1} p_{2}+k_{1} p_{2} m n_{1}+k_{2} p_{1} m n_{2},
$$

where $h_{0}$ is a linear polynomial in $x, y, z, w$. Thus $f(x, y, z, w)$ can be expressed as the required form (13).

In Eq.(13), the unknown coefficients $k_{1}, k_{2}$ and the unknown linear function $h_{0}$ can be solved by undetermined coefficients. Thus the implicit equation of a QSOB can be written as the determinant of a three by three matrix in which the entries are linear with $x, y, z, w$. The three by three matrix provides us a method to parameterize the cubic surface.

Let

$$
\left(L_{1}, L_{2}, L_{3}\right):=(s, t, 1) \cdot G,
$$

then $\left(L_{1}, L_{2}, L_{3}\right)$ can be rewritten as

$$
\left(L_{1}, L_{2}, L_{3}\right)=(x, y, z, w) \cdot H,
$$

where $H=\left(L_{i j}(s, t)\right)_{4 \times 3}$ with $L_{i j}(s, t) \in R[s, t] . L_{1}, L_{2}, L_{3}$ will serve as the weak $\mu$-basis of the parameterizaton of the cubic surface $f(x, y, z, w)=0$.

TheOREM 4.6. Let $L_{i j}(s, t)$ be given as above, and let $\mathbf{L}_{i}=\left(L_{1 i}, L_{2 i}, L_{3 i}, L_{4 i}\right)^{T}, i=1,2,3$. Then

$$
\mathbf{P}(s, t):=\left[\mathbf{L}_{1}, \mathbf{L}_{2}, \mathbf{L}_{3}\right]
$$

gives a quadratic parametrization of the cubic surface $f(x, y$, $z, w)=0$. Here $\left[\mathbf{L}_{1}, \mathbf{L}_{2}, \mathbf{L}_{3}\right]$ is the outer product of $\mathbf{L}_{1}, \mathbf{L}_{2}, \mathbf{L}_{3}$ i.e.,

$$
\begin{aligned}
& {\left[\mathbf{L}_{1}, \mathbf{L}_{2}, \mathbf{L}_{3}\right]:=} \\
& \left(\left|\begin{array}{lll}
L_{21} & L_{31} & L_{41} \\
L_{22} & L_{32} & L_{42} \\
L_{23} & L_{33} & L_{43}
\end{array}\right|,-\left|\begin{array}{lll}
L_{11} & L_{31} & L_{41} \\
L_{12} & L_{32} & L_{42} \\
L_{13} & L_{33} & L_{43}
\end{array}\right|,\right. \\
& \left.\left|\begin{array}{lll}
L_{11} & L_{21} & L_{41} \\
L_{12} & L_{22} & L_{42} \\
L_{13} & L_{23} & L_{43}
\end{array}\right|,-\left|\begin{array}{lll}
L_{11} & L_{21} & L_{31} \\
L_{12} & L_{22} & L_{32} \\
L_{13} & L_{23} & L_{33}
\end{array}\right|\right) \text {. }
\end{aligned}
$$

Proof. Without loss of generality, we assume that the self-intersection line $l$ coincides with $x$-axis, i.e., $l$ is defined by $y=z=0$.

We first prove that $\mathbf{L}_{1}, \mathbf{L}_{2}, \mathbf{L}_{3}$ are $R[s, t]$-linearly independent, i.e., the outer product of $\mathbf{L}_{1}, \mathbf{L}_{2}, \mathbf{L}_{3}$ is not a zero vector.

Consider two planes $p_{1}:=y-\lambda_{1} z=0$ and $p_{2}:=y-\lambda_{2} z=$ 0 passing through $x$ axis, here $\lambda_{1} \neq \lambda_{2}$. Then $f(x, y, z, w)=$ $\operatorname{det}(G)$ with

$$
G=\left(\begin{array}{ccc}
y-\lambda_{1} z & 0 & z \\
0 & y-\lambda_{2} z & z \\
h_{1} & h_{2} & h_{3}
\end{array}\right) .
$$

where $h_{1}=h_{11} x+h_{13} z+h_{14} w, h_{2}=h_{21} x+h_{23} z+h_{24} w$, $h_{3}=h_{31} x+h_{32} y+h_{33} z+h_{34}$, and $h_{i j}, i=1,2,3, j=1,2,3,4$ are constants. Furthermore, at least one of $h_{11}, h_{21}, h_{31}$ is nonzero.

From (14) and (15), we get

$$
H=\left(\begin{array}{ccc}
h_{11} & h_{21} & h_{31} \\
s & t & h_{32} \\
-\lambda_{1} s+h_{13} & -\lambda_{2} t+h_{23} & s+t+h_{33} \\
h_{14} & h_{24} & h_{34}
\end{array}\right)
$$

Thus

$$
\begin{aligned}
& \mathbf{L}_{1}=\left(h_{11}, s,-\lambda_{1} s+h_{13}, h_{14}\right), \\
& \mathbf{L}_{2}=\left(h_{21}, t,-\lambda_{2} t+h_{23}, h_{24}\right), \\
& \mathbf{L}_{3}=\left(h_{31}, h_{32}, s+t+h_{33}, h_{34}\right) .
\end{aligned}
$$

One can verify directly that $\left[\mathbf{L}_{1}, \mathbf{L}_{2}, \mathbf{L}_{3}\right]$ can't be identically the zero vector (otherwise $h_{11}=h_{21}=h_{31}=h_{14}=$ $h_{24}=h_{34}=0$, which implies that $f$ involves only $y, z$, an impossibility), and it thus gives a parametrization of the cubic surface. Furthermore, it is easy to see that the total degree of the parametrization is two. This completes the proof.

Based on the above theorem, we can devise an algorithm to compute a quadratic parametrization of a cubic surface with a self-intersection line.

\section{Algorithm PARAMETRIZATION-QSOB}

Input: The implicit equation $f(x, y, z, w)=0$ of a QSOB.

Output: The parametric equation of the QSOB.

Procedure:

1. Detect whether the cubic surface $f(x, y, z, w)=0$ has a self-intersection line or not by the algorithm SELFINTERSECTION-QSOB-II. If there exists a self-intersection line, compute it and denote it as $l$. Otherwise, Stop.

2. Choose any two distinct planes $p_{1}, p_{2}$ passing through the line $l$. Substituting the equation of each plane 
into $f(x, y, z, w)=0$, we will get a factorization as $m^{2} n_{1}$ and $m^{2} n_{2}$ respectively. Here $m, n_{1}, n_{2}$ are linear functions in $x, y, z, w$.

3. Solve for unknown coefficients $k_{1}, k_{2}, k_{3}, k_{4}, k_{5}, k_{6}$ from

$$
f(x, y, z, w)=\left|\begin{array}{ccc}
p_{1} & 0 & m \\
0 & p_{2} & m \\
k_{1} n_{1} & k_{2} n_{2} & k_{3} x+k_{4} y+k_{5} z+k_{6} w
\end{array}\right|
$$

4. Construct the weak $\mu$-basis as in the form (15). Compute the outer product of the weak $\mu$-basis. The result is a quadratic parametrization of the cubic surface $f(x, y, z, w)=0$.

We give an example to illustrate the above algorithm. 1 is

Example 2. The implicit equation of $\mathbf{P}(s, t)$ in Example

$$
\begin{aligned}
f(x, y, z, w):= & -x^{3}+3 x^{2} y+x^{2} z+3 x^{2} w \\
& -2 x z w-3 x w^{2}+z w^{2}+w^{3}=0 .
\end{aligned}
$$

and the singular locus of the cubic surface is $(x, y, z, w)=$ $(0, y, z, 0)$. Choosing the planes $x-w=0, x=0$ passing through the self-intersection line, we can get the following factorization of $f(x, y, z, w)$ :

$$
f(x, y, z, w)=\left|\begin{array}{ccc}
x-w & 0 & w \\
0 & x & w \\
-3 y & z+w & -x+3 y+z+2 w
\end{array}\right| .
$$

The weak $\mu$-basis is thus

$$
(s x-s w-3 y, t x+z+w,(s+t) w-x+3 y+z+2 w),
$$

or in vector form

$$
\begin{aligned}
& \mathbf{L}_{1}=(s,-3,0,-s), \quad \mathbf{L}_{2}=(t, 0,1,1), \\
& \mathbf{L}_{3}=(-1,3,1, s+t+2) .
\end{aligned}
$$

Thus the outer product of $\mathbf{L}_{1}, \mathbf{L}_{2}, \mathbf{L}_{3}$ gives a parametrization of the cubic surface:

$$
\mathbf{P}(s, t)=\left(-3 t-3,-s^{2}, 3 t^{2}-3 s+6 t+3,3 s-3 t-3\right) .
$$

Example 3. Consider the cubic surface

$$
f(x, y, z, w):=x z^{2}+y^{2} z+2 y^{2} w+y z w=0 .
$$

The self-intersection line of $f=0$ is $(x, y, z, w)=(x, 0,0, w)$. Choosing two planes $y-z=0, y=0$ passing through the self-intersection line, we obtain

$$
f(x, y, z, w)=\left|\begin{array}{ccc}
y-z & 0 & z \\
0 & y & z \\
-x-z-3 w & x & z+2 w
\end{array}\right| .
$$

The weak $\mu$-basis is

$$
\begin{aligned}
& \mathbf{L}_{1}=(-1, s,-s-1,-3), \quad \mathbf{L}_{2}=(1, t, 0,0), \\
& \mathbf{L}_{3}=(0,0, s+t+1,2) .
\end{aligned}
$$

Thus the parametrization of the cubic surface is given by $\mathbf{P}(s, t)=\left(-s t-3 t^{2}-t, s+3 t+1,-2 s-2 t, s^{2}+2 s t+t^{2}+s+t\right)$.

\section{CONCLUSION}

In this paper, we have unified the implicitization and parametrization of a quadratic surface with one simple base point (QSOB) by using the weak $\mu$-basis of the QSOB. Simple and efficient algorithms are derived to realize the conversion between implicit form and parametric form. We also provide a method to detect and compute the self-intersection line of a QSOB, either in parametric form or in implicit form. The singular locus of the QSOB is important for the computation of the weak $\mu$-basis of a cubic surface derived from a QSOB. Examples are provided to illustrate the algorithms.

It would be interesting to generalize the moving plane method to handle the conversion between implicit form and parametric form of higher degree surfaces.

\section{ACKNOWLEDGMENTS}

This work is supported by A National Key Basic Research Project of China (No. 2004CB318000), NSF of China (No. 10671192, 60225002 and 60533060), One hundred Talent Project supported by CAS, Specialized Research Fund for the Doctoral Program of Higher Education (No. 20060358055), the 111 Project (No. b07033).

\section{REFERENCES}

[1] C. Bajaj, R. Holt, and A. Netravali, Rational parametrizations of nonsingular real cubic surfaces, ACM Transactions on Graphics, Vol.17, 1-31, 1998.

[2] T. G. Berry and R. R. Patterson, Implicitization and parametrization of nonsingular cubic surfaces, Computer Aided Geometric Design, Vol.18, 723-738, 2001.

[3] B. Buchberger, Applications of Groebner bases in nonlinear computational geometry, In Kapur, D. and Mundy, J., (eds), Geometric Reasoning, Elsevier Science Publisher, MIT Press, 413-446, 1989.

[4] L. Busé, D. Cox, and C. D'Andrea, Implicitization of surfaces in $\mathbb{P}^{3}$ in the presence of base points, Journal of Algebra and Its Applications, Vol.2, 189-214, 2003.

[5] F. Chen, J. Zheng, and T. W. Sederberg, The $\mu$-basis of a rational ruled surface, Computer Aided Geometric Design, Vol.18, 61-72, 2001.

[6] F. Chen and W. Wang, Revisiting the $\mu$-basis of a rational ruled surface, Journal of Symbolic Computation, Vol.36, No.5, 699-716, 2003.

[7] F. Chen and W. Wang, Computing real inflection points of cubic algebraic curves, Computer Aided Geometric Design, Vol.20, 101-117, 2003.

[8] F. Chen, D. Cox, and Y. Liu, The $\mu$-basis and implicitization of a rational parametric surface, Journal of Symbolic Computation, Vol.39, 689-706, 2005.

[9] E. Chionh and R. Goldman, Using multivariate resultants to find the implicit equation of a rational surface, The visual Computer: International Journal of Computer Graphics, Vol.8, 171-180, 1992.

[10] A. Coffman, A.J. Schwartz, and C. Stanton, The algebra and geometry of Steiner and other quadratically parametrizable surfaces, Computer Aided Geometric Design, Vol.13, 257-286, 1996. 
[11] D. A. Cox, D. Little, and D. O'Shea, Ideals, Varieties, and Algorithms - An Introduction to Computational Algebraic Geometry and Commutative Algebra, 2nd ed., Springer, 2005.

[12] D. A. Cox, T. W. Sederberg, and F. Chen, The moving line ideal basis of planar rational curves, Computer Aided Geometric Design, Vol.15, 803-827, 1998.

[13] J. Deng, F. Chen, and L. Shen, Computing $\mu$-bases of rational curves and surfaces using polynomial matrix factorization, Proceedings of the ISSAC'2005, Manuel Kauers ed., ACM Press, USA, 132-139, 2005.

[14] B. Hunt, The geometry of some special arithmetic quotients, Lecture Notes in Mathematics 1637, Springer, 1996.

[15] P. Irene, Theory and history of geometric models, Academic Press Europe, 41-42, 2007.

[16] T. W. Sederberg, D. Anderson, and R. Goldman, Implicit representation of parametric curves and surfaces, Computer Vision, Graphics, and Image Processing, Vol.28, 72-84, 1984.
[17] T. W. Sederberg and F. Chen, Implicitization using moving curves and surfaces, Proceedings of Siggraph'1995, 301-308, 1995.

[18] T. W. Sederberg, Techniques for cubic algebraic surfaces, Part 2. IEEE Computer Graphics and Applications, Vol.10, No.5, 12-21, 1990.

[19] J. Semple and L. Roth, Introduction to Algebraic Geometry, Clarendon Press, Oxford, Reprint of the 1949 original, 1985.

[20] J. Schicho, Rational parametrization of real algebraic surfaces, ISSAC'98: Proceedings of the 1998 international symposium on Symbolic and algebraic computation, ACM Press, 302-308, 1998.

[21] L. Shen, F. Chen, and J. Deng, Implicitization and parametrization of quadractic and cubic surfaces by $\mu$-bases, Computing, Vol.5, 131-142, 2006.

[22] W. Wang, B. Joe, and R. Goldman, Computing quadric surface intersections based on an analysis of plane cubic curves, Graphical Models, Vol.64, 335-367, 2002. 